\title{
榕小蜂的产卵模式及其对子代性比的影响
}

\author{
孙宝发 ${ }^{1,2}$, 王瑞武 ${ }^{1, *}$, 胡 忠 ${ }^{3, *}$, \\ (1. 中国科学院昆明动物研究所 遗传资源与进化国家重点实验室, 生态学与环境保护中心, 云南 昆明 650223 ; \\ 2. 中国科学院动物研究所 动物进化与系统学院重点实验室, 北京 $100101 ; 3$. 汕头大学 生物系, 广东 汕头 515063)
}

\begin{abstract}
摘要: 于 2007 年 4 月-2007 年 7 月在中国科学院西双版纳热带植物园, 通过干预控制榕小蜂产卵的方法研 究了聚果榕的传粉榕小蜂 Ceratosolen fusciceps 和非传粉小蜂 Platyneura mayri 及垂叶榕的非传粉小蜂 Acophila sp.1 和 Wakerella benjamini 等的产卵顺序。对传粉榕小蜂, 在完成放蜂后不同间隔时间向榕果内注入乙醚杀死小 蜂; 对非传粉榕小蜂, 在放蜂后不同间隔时间将其从纱网袋内全部放出, 从而控制了各种榕小蜂的产卵时间。到 榕果成熟后, 收集了榕果内的小蜂, 并分析比较各种榕小蜂在不同产卵时间下的子代性比。结果表明: Ceratosolen fusciceps、P. mayri 和 Acophila sp.1 在产卵的最初时间内倾向于产下更多的雄性后代，而随后的时间内则产下更多 的雌性伴随少量的雄性后代, 这样的产卵顺序导致子代性比随着母代产卵时间的延长而下降, 榕小蜂后代雌性比 例显著高于雄性。同时, 子代榕小蜂数量随母代产卵时间的延长而增加, 这在一定程度上解释了单头繁殖雌蜂的 子代性比随子代数量的增加而减少的现象。而 Wakerella benjamini 在产卵顺序上是随机的, 在其开始产卵后的不 同时间段内子代性比都接近于 $50 \%$ 。这一结果表明榕小蜂的产卵顺序与母代产卵时间的长短对子代性比有极为重 要的影响。
\end{abstract}

关键词: 榕小蜂; 性比; 产卵顺序; 局域交配竞争; 偏雌性比

中图分类号: Q969.54; Q964 文献标识码: A 文章编号: 0254-5853-(2009)05-0559-06

\section{Ovipositing Pattern of the Fig Wasps and Its Effect on the Offspring Sex Ratio}

\author{
SUN Bao-fa ${ }^{1,2}$, WANG Rui-wu ${ }^{1, *}$, HU Zhong ${ }^{3,{ }^{*}}$ \\ (1. Ecology, Conservation, and Environment Center, State Key Laboratory of Genetic Resources and Evolution, Kunming Institute of Zoology, the Chinese \\ Academy of Sciences, Kunming, Yunnan 650223, China; 2. Key Laboratory of Zoological Systematics and Evolution, CAS, Institute of Zoology, Chinese \\ Academy of Sciences, Beijing 100101, China; 3. Biology Department, Shantou University, Shantou, Guangdong 515063, China)
}

\begin{abstract}
In this study, we experimentally examined the ovipositing sequence of the pollinator Ceratosolen fuscicep, non-pollinating wasp Platyneura mayri of Ficus racemosa and non-pollinating wasp Acophila sp.1 and Wakerella benjamini of Ficus benjamina in Xishuangbanna from Apr. 2006 to Jun. 2007. For the pollinating fig wasps, we injected ether into the receptive fruits with different time intervals after introducing the fig wasps, killing the fig wasps; for the non-pollinating fig wasps, we manually controlled ovipositing time length through organdy bags. After the treated fruits developed to be mature stage, we collected the adult wasps, and then analyzed and compared the offspring sex ratio under different ovipositing time length. The data showed that fig wasp C. fuscicep, P. mayri and Acophila sp.1 firstly oviposit their male offspring. The male offspring was much higher than female, if the mother wasps only oviposited one hour, whist the female offspring will be increased with the increase of oviposting time of mother wasps and the female offspring will be much higher than male offspring when the mother wasps could sufficiently oviposit their offspring in these three fig wasps. These results partly explain that the female offspring sex ratio decrease with increase of number of foundresses. However, the ovipositing sequence of Wakerella benjamini randomly oviposit their eggs and the sex ratio is about 1:1. Our results here imply that the ovipositing sequence and ovipositing time length of mother wasps might be of the most important factors determining the offspring sex ratio of fig wasps.
\end{abstract}

收稿日期: 2009-04-29; 接受日期: 2009-09-07

基金项目：国家自然科学基金资助项目(30670272,30770500); 国家重点基础研究发展计划（“973”计划）(2007CB411600); 云南省科技 厅项目 (20080A001); 云南省自然科学基金（2009CD104）

“通讯作者 (Corresponding authors), E-mail: wangrw@mail.kiz.ac.cn; hu@stu.edu.cn 第一作者简介: 孙宝发 (1983-), 男, 汉族, 河北唐山人, 博士生, 主要从事进化生态学研究 
Key Words: Fig wasp; Sex ratio; Oviposition sequence; Local mate competition; Female biased sex ratio

性比理论是进化生物学的研究热点之一。在随 机交配的种群中亲代在子代雌性和雄性之间的基 因投资应是相等的, 只有 $1: 1$ 的性比率在进化上才 是稳定的 (Fisher, 1930)。而Hamilton (1967, 1979) 则认为近亲交配将会导致物种的偏雌性演化, 并认 为榕小蜂将为他的理论推理提供验证。因此, 榕小 蜂一直被作为检验性分配理论的模式材料 (West et a1, 2001; Weiblen, 2002)。

与其它膜翅目昆虫一样, 榕小蜂的性别决定系 统是单双倍体 (haplo-diploid) 类型, 即雌蜂是由受 精卵发育而来, 是二倍体, 具有两套染色体; 雄蜂 则由未受精的卵发育形成, 为单倍体, 只有一套染 色体 (Flanders, 1956; Peng et al, 2005)。Herre (1987) 认为榕小蜂能通过控制卵是否受精来调整子代性 比 (性比=子代雄蜂数量/子代小蜂总量), 受精的卵 发育为双倍体的雌蜂, 而未受精的卵发育为单倍体 的雄蜂。而榕小蜂在产卵时, 它先产雄性还是先产 雌性或者随机产生雌、雄子代及其对子代性比影响 的研究结果并不一致。Kathuria et al (1999)的研究 结果表明, 在母代榕小蜂的不同产卵时间内, 其子 代性比无明显差异; 而 Shazia et al (2008)的结果则 表明, 产卵时间对其子代性比有很大的影响。在榕 小蜂的产卵效率大致相同的情况下，不同产卵时间 下榕小蜂产下的雌雄子代数量与其产卵顺序有关。 然而, 到目前为止, 榕小蜂在榕果内的产卵顺序并 不十分清楚。本研究通过对西双版纳广泛分布的聚 果榕的传粉榕小蜂 Ceratosolen fusciceps 和非传粉 榕小蜂 Platyneura mayri, 以及垂叶榕上寄居的非传 粉榕小蜂 Acophila sp.1 和 Wakerella benjamini 进行 放蜂实验来分析其产卵顺序。

\section{1 材料与方法}

\section{1 研究材料}

聚果榕 ( Ficus racemosa) 传粉榕小蜂 Ceratosolen fusciceps、非传粉小蜂 Platyneura mayri 和垂叶榕 (Ficus benjamina) 非传粉小蜂 Acophila sp.1、Wakerella benjamini。Ceratosolen fusciceps 属 于 Agaonidae 科, 整个身体呈黑色, 翅膀长于身体, 在榕果内产卵, 传粉小蜂。Platyneura mayri 属于 Sycophaginae 科, 体色棕黄, 产卵器约 3 倍体长, 在传粉榕小蜂产卵前后从果外产卵, 造瘘者。
Acophila sp.1 属于 Epichrysomallinae 科, 雌蜂体黑 色, 雄蜂有翅。Walkerella benjamini 属于 Otitesellinae 科, 雌蜂体呈铜绿色, 产卵器短, 雄蜂无翅。Acophila sp. 1 和 Walkerella benjamini 均为造瘘者, 在传粉榕 小蜂产卵前从果外产卵。

\section{2 研究方法}

我们于 2007 年 4 月 -2007 年7月在西双版纳预 仑镇选取两种样树各 1 株进行了定量放蜂实验。在 果实刚萌发花芽的时候用 120 篮目的绢纱袋进行套 袋, 待果实发育到各种小蜂的产卵期时按小蜂种类 分别进行放蜂, 并通过不同的处理方法达到控制小 蜂产卵时间的目的。对传粉榕小蜂, 在完成放蜂后 间隔不同时间向榕果内注入乙醚杀死小蜂; 对非传 粉榕小蜂, 在放蜂后间隔不同时间将其从袋内全部 放出, 从而达到了控制小蜂产卵时间的目的, 并分 析榕小蜂在不同产卵时间下其子代性比是否存在 差异。当隐头果发育成熟时, 采下单果放入绢纱袋 内带回实验室, 让子代榕小蜂自然羽化在隔离袋 内。解剖成熟果, 收集每个单果内所有的榕小蜂后 代, 并统计雌雄数量。

1.2.1 聚果榕传粉小蜂放蜂果注入乙醚实验 首 先通过预实验来确定榕果的乙醚注入量。当聚果榕 样树果实刚萌发花芽的时候用 120 篮目的绢纱袋 $(20 \mathrm{~cm} \times 20 \mathrm{~cm}$ ) 隔离 100 个左右刚结的隐头果。当 榕果进入雌花期时选择果径相近的榕果进行传粉 小蜂的放蜂实验, 每果均同时放入 3 只小蜂。放蜂 后用注射器吸取不同量的乙醚通过榕果顶端的苞 片口向榕果内注入, 注入后隔 $3 \mathrm{~min}$ 将榕果剖开, 观察榕小蜂是否被乙醚杀死。通过反复实验确定 $0.05 \mathrm{~mL}$ 乙醚的注入量可以将榕小蜂完全杀死, 且 由于乙醚的挥发性很强, 注入 $0.05 \mathrm{~mL}$ 乙醚的榕果 在发育过程中并不会发生脱落, 能够正常发育至成 熟。因此, 在聚果榕传粉小蜂放蜂后间隔不同时间 向榕果内注入乙醚将传粉小蜂杀死可以达到控制 其产卵时间的目的。不注射乙醚的榕果内的传粉小 蜂产卵时间, 我们通过对将放完蜂的榕果在不同的 间隔时间后剖开, 发现小蜂在果内的平均寿命为

(24.32 \pm 3.56$) \mathrm{h}(\mathrm{Mean} \pm S D)$, 因此我们可以将不 注乙醚果内的传粉小蜂的产卵时间记为 $24 \mathrm{~h}$ 。

用 120 笁目的绢纱袋 $(20 \mathrm{~cm} \times 20 \mathrm{~cm})$ 对同一样 树的榕果进行套袋隔离。当榕果进入雌花期时进行 
传粉小蜂的控制放蜂实验。选择与预实验中放蜂果 果径相近的 120 个套袋榕果, 将其平均分为 6 个组, 每组 20 个果, 每果均同时放入 3 只小蜂。放蜂完 成后, 重新套上隔离袋。第 1 组放蜂后不注乙醚, 其余组分别在放蜂后间隔 $1 、 2 、 4 、 6 、 8 \mathrm{~h}$ 注入 0.05 $\mathrm{mL}$ 乙醚, 将果内小蜂杀死。当果实发育成熟时, 将单果分袋采回, 让子代榕小蜂自然羽化在隔离袋 内。解剖成熟果, 收集每个单果内所有的榕小蜂后 代, 并统计雌雄数量。

1.2.2 非传粉小蜂的放蜂隔离实验 当聚果榕和 垂叶榕样树果实刚萌发花芽的时候用 120 篮目的绢 纱袋 $(20 \mathrm{~cm} \times 20 \mathrm{~cm})$ 对刚结的隐头果进行隔离: 聚果 榕隔离 120 个果, 垂叶榕隔离 240 个果。当榕果发 育到各种非传粉小蜂的产卵期时, 选取处于成熟期 的聚果榕和垂叶榕的榕果让其自然出蜂, 按小蜂种 类进行收集, 分别对聚果榕进行 P. mayri, 对垂叶 榕进行 Acophila sp.1 和 Wakerella benjamini 的单果 隔离放蜂实验, 每果放蜂量均为 20 只, 每种小蜂 各放 120 个放蜂果。单果放蜂完成后, 继续套上隔 离袋, 将每种小蜂的 120 个放蜂果平均分为 6 个组, 第 1 组在放蜂后不再将非传粉小蜂放出, 其余 5 组 分别在放蜂后 1、2、4、6、8 h 后用空的纱网袋换 下重新套上, 直到隐头果发育成熟。在雨季, $P$. mayri、Acophila sp.1 和 Wakerella benjamini 在果外 产卵的平均时间均为 $1 \mathrm{~d}$ 左右, 因此, 我们将其不 再放出纱网袋的小蜂的产卵时间均记为 $24 \mathrm{~h}$ 。果实 发育成熟时将单果分袋采回, 让子代榕小蜂自然羽 化在隔离袋内。解剖成熟果, 收集每个单果内所有 的榕小蜂后代, 并统计雌雄数量。

\section{3 数据分析}

通过非参数检验对数据是否属于正态分布进 行了检验, 通过独立样本 $T$-test、单因素方差分析 (ANOVA)、Mann-Whitney $U$ tests、LSD多重比较等 方法对各种小蜂在不同产卵时间下的子代性比进 行比较, 采用Pearson相关分析等方法分析母代产卵 时间与子代小蜂性比之间的相关性。所有计算分析 均通过调用SPSS 13.0软件包程序实现。文中图表制 作主要是通过调用Excel软件包程序完成。

\section{2 结 果}

\section{1 聚果榕传粉榕小蜂子代性比与母代产卵数量 相关}

聚果榕传粉榕小蜂Ceratosolen fusciceps放蜂果
在放蜂后间隔不同时间注入乙醚处理，其成熟果内 的传粉小蜂子代性比如图1所示。

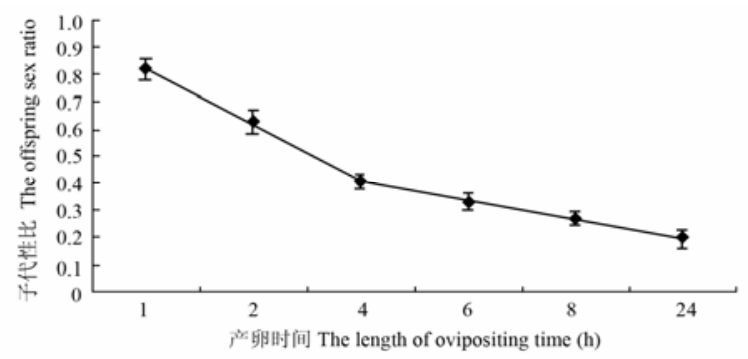

图 1 不同产卵时间下传粉榕小蜂Ceratosolen fusciceps 的子代性比

Fig.1 Offspring sex ratio of pollinator Ceratosolen fusciceps under different ovipositing time length

通过LSD多重比较可以发现，母代在不同产卵 时间下所产生的子代小蜂性比存在着显著性差异 $\left(F_{(5,114)}=1371.044, P<0.001\right)$ 。由图1可以看出, 子代小蜂性比会随着母代产卵时间的增加而急剧 下降。母代产卵时间和子代小蜂性比呈显著负相关 $(n=120, r=-0.635, P<0.001)$ 。母代在不同产卵时 间内所产生的子代雌雄数量如图2所示。

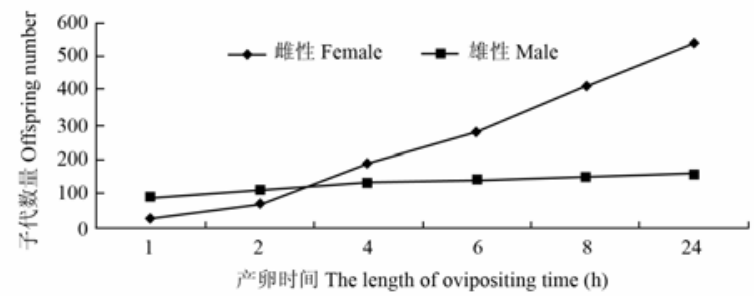

图 2 不同产卵时间下子代传粉榕小蜂Ceratosolen fusciceps的雌雄数量

Fig. 2 The number of female and male offspring of pollinator Ceratosolen fusciceps under different ovipositing time length

从图 2 可以看出, 子代雌性小蜂的数量, 是随 着产卵时间的增加而显著上升，两者之间有着显著 的正相关关系 $(n=120, r=0.762, P<0.001)$ ，且不 同产卵时间下各组之间, 其数量存在着显著性差异 $(F(5,114))=648.366, P<0.001)$ 。而子代雄蜂数量 虽然也随着母代产卵时间的增加呈上升趋势, 但子 代雌性数量随母代产卵时间的延长而增加的速度 显著快于子代雄性数量。因此, 子代性比随母代产 卵时间的增加而显著降低。在母代开始产卵的最初 $1 \mathrm{~h}$ 内, 子代雄蜂的数量远高于子代雌蜂数量。而 在母代产卵的随后几个小时内, 雌蜂数量却一直呈 增加的趋势, 而子代雄蜂增加的数量远小于雌蜂。 
这表明, 传粉榕小蜂在产卵的顺序上有明显的倾向 性, 即倾向于先产下更多的雄性。因此, 当小蜂的 产卵时间仅为 $1 \mathrm{~h}$ 的条件下, 在子代小蜂中雄性占 据了大多数。而随着产卵时间延长, 母代则倾向于 产下更多的雌性。产卵时间的延长导致了产卵数的 增加, 因此表现为子代性比随子代数量的增加而显 著降低。

\section{2 聚果榕非传粉小蜂 P. mayri 子代性比与母代 产卵时间相关}

非传粉小蜂P. mayri在放蜂后间隔不同时间将 其放出纱网袋, 其子代小蜂性比也因母代产卵时间 的不同而呈现显著差异 $\left(F_{(5,114)}=541.151, P<0.001\right)$ 。 与传粉小蜂一样, P. mayri的性比也随着母代产卵 时间的增加而急剧下降 (图3)。子代小蜂的性比和 母代产卵时间也呈显著的负相关关系 $(n=114$, $r=-0.634, P<0.001$ )。比较不同产卵时间下的雌雄 小蜂数量, 发现与聚果榕传粉小蜂各组性比存在差 异的原因相同, P. mayri在产卵的顺序上也是倾向 于先产雄性, 所以小蜂的产卵时间越短, 性比就会 越高, 而随着产卵时间的增加, 子代小蜂性比因雌 蜂的显著增加而急剧下降。

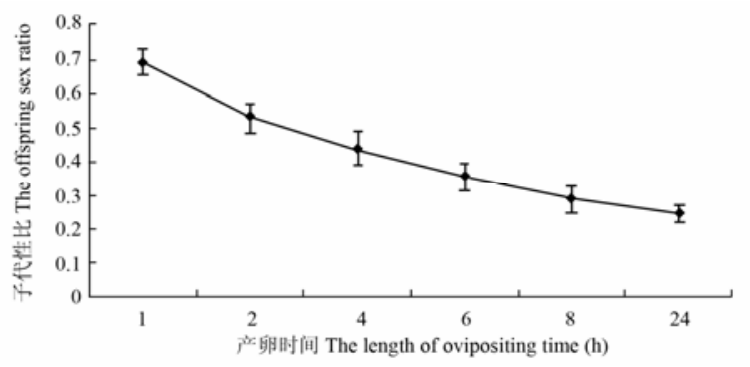

图 3 不同产卵时间下P. mayri的子代小蜂性比

Fig. 3 Offspring sex ratio of $P$. mayri under different ovipositing time length

\subsection{Acophila sp. 1 性比与产卵时间有关, Wakerella benjamini 性比与产卵时间无关}

垂叶榕非传粉小蜂 Acophila sp.1 和 Wakerella benjamini 在不同的隔离时间下子代小蜂的性比如 图 4 所示。

Acophila sp.1子代性比与聚果榕的传粉小蜂和 非传粉小蜂P. mayri的结果相一致, 在放蜂后间隔 不同时间再将其放出后子代小蜂的性比因母代产 卵时间的不同而出现显著差异 $(F(5,114)=189.791$, $P<0.001$ )。它的性比也随着母代产卵时间的增加而 急剧下降 (图4)。对母代产卵时间和子代小蜂性比 的相关分析结果表明: 子代小蜂性比和母代产卵时
间也呈显著负相关 $(n=120, r=-0.671, P<0.001)$ 。 而Wakerella benjamini的性比却与聚果榕传粉小蜂 和非传粉小蜂P. mayri 以及Acophila sp.1存在明显 差异: 母代产卵时间对子代性比无显著影响 $\left(F_{(5,114)}\right.$ $=0.899, P>0.05)$ ，母代在不同产卵时间下，其子代 小蜂的性比均为 $50 \%$ 左右, 小蜂产卵时间和子代性 比无显著相关性 $(n=120, r=-0.072, P>0.05)$ 。这 表明, 当Wakerella benjamini母代产卵时, 它产下雌 雄小蜂的顺序是随机的, 并没有明显的倾向性。

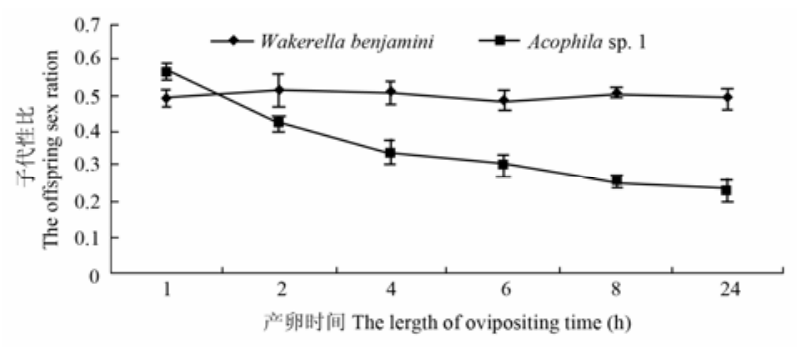

图 4 不同产卵时间下Acophila sp.1和Wakerella benjamini 的子代小蜂性比

Fig. 4 Offspring sex ratio of Acophila sp.1 and Wakerella benjamini under different ovipositing time length

\section{3 讨 论}

\section{1 母代小蜂的产卵时间影响子代性比}

我们的研究结果表明, 榕小蜂的产卵模式对子 代性比有着重要的影响。聚果榕传粉小蜂和非传粉 小蜂P. mayri 以及垂叶榕的非传粉小蜂Acophila $\mathrm{sp}$. 1 在产卵顺序上都有着明显的倾向性。它们在产 卵的早期都倾向于多产雄蜂, 而随着产卵时间的延 长, 子代雌蜂的产卵量显著增加, 而子代雄蜂增加 的量远小于雌性子代。这与Shazia et al（2008）的 结论相一致; 而垂叶榕非传粉小蜂 Wakerella benjamini的产卵模式则存在差异, 即随机地产下雌 雄后代，导致其性比接近 $50 \%$ 。这表明不同种类榕 小蜂在产卵顺序上存在着差异，母代产卵时间的长 短对子代性比的影响不一。先前的针对少数种类榕 小蜂进行研究所得出的结论是不可靠的(Kathuria et al, 1999; Shazia et al, 2008)。

在子代性比的调节上, 当榕小蜂先产雄性卵 时, 母代小蜂的产卵时间将影响到子代性比, 而榕 小蜂的产卵时间长短与其寿命相关。因此, 榕小蜂 的寿命长短可能是影响子代性比的因素之一。榕小 蜂在不同季节或不同的环境条件下, 其寿命长短有 着很大的差异 (Wang et al, 2005), 如温度和湿度条 
件就对榕小蜂寿命长短有着很大影响 (Wang et al, 2005; Dunn et al, 2008)。以往的研究结果表明, 榕 小蜂的寿命与湿度有着密切关系, 湿度越大, 榕小 蜂的寿命越长 (Dunn et al, 2008)。同时, 我们通过 观测发现榕小蜂在气温较高 (大于 $30^{\circ} \mathrm{C}$ ) 时通常仅 能存活 $1 \mathrm{~d}$ 左右, 而在气温较低 $\left(20^{\circ} \mathrm{C}\right.$ 左右 $)$ 时能存 活 $3 \mathrm{~d}$ 左右。榕小蜂的存活时间越长, 其产卵时间也 随之延长, 子代性比随着其产卵量的增加而下降。 因此, 我们在考虑榕小蜂子代性比之时, 应综合考 虑到环境条件如温度湿度等对榕小蜂个体寿命的 影响。

\section{2 子代小蜂数量增加时其性比降低受母代产卵 模式的影响}

先前的对传粉榕小蜂的研究结果表明, 当单头 雌蜂在单果内繁殖时, 随着子代数量增加, 其性比 降低 (Hamilton, 1979; Frank, 1985; Greeff, 2002)。 同时, 在产卵的母代小蜂数量, 即Foundress确定的 情况下, Clutch size 即总花数(代表产卵场所的多少) 对子代小蜂性比的影响表现为子代性比随Clutch size的增加而减少。West et al（1998）对17种非传 粉小蜂自然种群的研究指出当单头繁殖雌蜂在一 个榕果上产卵时, 子代性比与子代数量也呈显著负 相关关系。传粉小蜂和非传粉小蜂的子代性比与子 代数量的负相关在一定程度上可能与小蜂的产卵 顺序相关。在本研究中, 当控制放一定数量的传粉 小蜂Ceratosolen fusciceps和非传粉小蜂Platyneura mayri和Acophila sp.1在一个榕果上产卵时, 子代数 量随着母代产卵时间的延长而增加。而雌性子代增 加的程度远高于雄性, 从而使子代性比与子代数量 呈显著的负相关关系, 这一结果在支持前人的基础 上一定程度地解释了Clutch size对子代性比影响的 原因, 即小蜂的产卵顺序可能是其原因之一。

\section{3 小蜂的产卵模式决定了母代寿命和相互干扰 对子代性比的影响}

经典性别演化理论认为局域交配竞争 ( LMC) 和近亲交配将会导致小蜂后代中雄性的比例随产 卵蜂数量的增加而增加, 当产卵蜂数比较多, 而接 近于随机交配时 (产卵蜂数量在9只以上), 雌雄比 例将接近1：1（Hamilton 1967; Herre 1985，1987)。 然而, 我们的实验结果显示: 榕小蜂的性别比例也
可能与其个体寿命的长短相关。因为榕小蜂的寿命 长短会影响到产下的子代数量, 而子代数量与其性 比有一定的相关性。在不同的环境条件或小蜂的个 体存在活力差异时, 相同进蜂量下小蜂的子代性比 也并不完全一致，甚至可能存在较大差异。同时， 不同种类榕小蜂的产卵顺序并不一致, 这使得小蜂 的性比问题更加复杂。产卵顺序存在差异的具体原 因到目前为止尚不明确, 有待于进一步研究。

在产卵时倾向于先产雄性的榕小蜂, 其母代个 体数量的增加导致子代性比上升的现象除了局域 交配竞争和近亲交配机制的作用外(Herre，1985; Hamilton, 1979; Herre et al, 1997), 资源的限制和榕 小蜂同时产卵时的相互干扰也发挥着一定的作用

(Wang et al, 2008)。当产卵的母代小蜂数量较少 时, 雌花资源对于榕小蜂来说是充足的, 而且榕小 蜂之间彼此干扰的情况也较少发生, 每只榕小蜂都 能产下尽可能多的卵; 而当母代小蜂数量增加时, 雌花资源的限制以及榕小蜂之间的彼此干扰导致 母代小蜂可能在刚产下一部分卵甚至产下的大部 分是雄性卵之后就终止了产卵, 这样子代雌性小蜂 的数量因为资源限制和干扰效应的作用而降低, 从 而导致了小蜂性比的上升。

母代小蜂的产卵顺序影响到子代性比, 且不同 榕小蜂的产卵顺序存在着差异。这一现象的发现说 明了榕小蜂的产卵模式对其性比有着重要的影响。

因为榕小蜂的产卵模式并不完全一致, 而且性比问 题本身也是很复杂的。早期的研究认为, 榕小蜂呈 现出偏雌的性比率, 主要是单双倍性别决定系统、 局域配偶竞争和近交效应共同调节的结果 (Hamilton, 1979)。传粉榕小蜂的乱交 (Peng et a1, 2005 ) 和非传粉榕小蜂的存在 (Pereira \& Prado, 2005;Peng et a1, 2005）也都会影响传粉榕小蜂的性 比率。本文仅对聚果榕的传粉小蜂 Ceratosolen fusciceps和非传粉小蜂P. mayri以及垂叶榕的两种 非传粉小蜂Acophila sp.1和Wakerella benjamini作为 代表来进行研究, 在其它榕小蜂中的结论可能有所 差异, 只有进一步增加研究材料, 对其它种榕小蜂 进行全面研究, 才能客观反应出产卵模式在性比调 控中的作用。 


\section{参考文献:}

Dunn DW, Yu DW, Ridley J, Cook JM. 2008. Longevity, early emergence and body size in a pollinating fig wasp - implications for stability in a fig-pollinator mutualism[J]. J Anim Ecol, 77: 927-935.

Fisher RA. 1930. The Genetical Theory of Natural Selection[M] Oxford:Oxford University Press.

Flanders SE. 1956. The mechanism of sex-ratio regulation in the (parasitic) Hymenoptera[J]. Insect Soc, 3: 325-334.

Frank SA. 1985. Hierarchical selection theory and sex ratios: On applying the theory and a test with fig wasps[J]. Evolution, 39: 949-964.

Greeff JM. 2002. Mating system and sex ratios of a pollinating fig wasp with dispersing males[J]. Proc $R$ Soc Lond B, 269: 2317-2323.

Hamilton WD. 1967. Extraordinary sex ratios[J]. Science, 156: 477-488.

Hamilton WD. 1979. Wingless and Fighting Males in Fig Wasps and Other Insects[M]//Blum MS, ed. Sexual Selection and Reproductive Competition in Insects London: Academic Press.

Herre EA, West SA, Cook JM, Compton SG, Kjellberg F. 1997. Fig-associated Wasps: Pollinators and Parasites, Sex-ratio Adjustment and Male Polymorphism,Ppopulation Structure and its Consequences [M] // Choe JC , Crespi BJ eds. The Evolution of Mating Systems in Insects and Arachnids. Cambridge: Cambridge University Press, 226-239.

Herre EA. 1987. Optimality, plasticity and selective regime in fig wasp sex ratios[J]. Nature, 329: 627-629.

Herre EA. 1985. Sex ratio adjustment in fig wasps[J]. Science, 228: 896-898.
Kathuria P, Compton SG, Ganeshaiah KN, Greeff JM. 1999. What fig wasp sex ratios may or may not tell us about sex allocation strategies[J]. Oikos, 87: 520-530.

Peng YQ, Yang DR, Wang QY. 2005. Adjustment and stabilization of sex ratio in Ceratosolen solmsi marchali[J]. Acta Ecol Sin, 6: 1347-1351.[彭艳琼, 杨大荣, 王秋燕. 2005. 对叶榕传粉小蜂性比率 的调节和稳定. 生态学报, 6: 1347-1351.]

Pereira RAS, do Prado AP. 2005. Non-pollinating wasps distort the sex ratio of pollinating fig wasps[J]. Oikos, 110: 613-619.

Shazia R, Nazia S, Stephen GC, Jamie CM. 2008. The mechanism of sex ratio adjustment in a pollinating fig wasp[J]. Proc $R$ Soc Lond $B, 275$ : 1603-1610.

Wang RW, Yang JX, Yang DR. 2005. Seasonal changes in the trade-off among the fig-supported wasps and viable seeds in figs and their evolutionary implications[J]. J Integr Plant Biol, 47(2): 144-155.

Wang RW, Shi L, Ai SM, Zheng Q. 2008. Trade-off between the reciprocal mutualists: local resource availability oriented interaction in fig/fig wasp mutualism[J]. J Anim Ecol, 77: 616-623.

Weiblen GD. 2002. How to be fig wasp[J]. Annu Rev Entomol, 47: 229-330. West SA, Murray MG, Machado CA, Griffin AS, Herre EA. 2001. Testing Hamilton's rule with competition between relatives[J]. Nature, 409: 510-513.

West SA, Herre EA. 1998. Partial local mate competition and the sex ratio: A study on non-pollinating fig wasps[J]. J Evol Biol, 11: 532-548.

\section{聊城大学农学院 “动物生态生理学” 实验室简介}

聊城大学农学院 “动物生态生理学” 实验室依托聊城大学农学院实验中心和山东省高校生态学与生物多 样性重点实验室, 主要开展小型哺乳动物的能量学研究, 即从能量学角度深入探讨了小型哺乳动物对自然环 境的适应策略和生理调节机理。实验室负责人赵志军博士于2006年7月毕业于中国科学院动物研究所。2006 年7月赴俄罗斯科学院生态与进化研究所（Institute of Ecology and Evolution, Russian Academy of Science）学 术交流和合作研究；2007.5-2008.5 赴英国阿伯丁大学生命科学与医学学院（College of Life Science and Medicine, University of Aberden）作博士后研究。承担英国皇家学会课题1项、国家自然基金1项、聊城大学 科研启动基金1项；参与山东省教育厅课题1项、企业横向课题1项。

\section{赵志军}

